PROCEEDINGS OF THE

AMERICAN MATHEMATICAL SOCIETY

Volume 139, Number 11, November 2011, Pages 4137-4141

S 0002-9939(2011)10769-X

Article electronically published on February 21, 2011

\title{
REPRESENTING POINTSETS AS UNIONS OF BOREL SETS
}

\author{
HOWARD BECKER
}

(Communicated by Julia Knight)

\begin{abstract}
We consider a method of representing projective sets by a particular type of union of Borel sets, assuming AD.
\end{abstract}

\section{The Main Theorem}

The axiom of determinacy (AD) is always assumed.

The basic references for descriptive set theory and AD are Jackson 3 and Moschovakis [5], to which we refer the reader for unexplained terminology, concepts and theorems. The proofs in this paper are written for a reader with a good understanding of this topic.

$\mathcal{B}$ denotes the class of Borel subsets of $\mathcal{N}\left(=\omega^{\omega}\right) \cdot \gamma_{2 n+1}$ denotes the predecessor of $\boldsymbol{\delta}_{2 n+1}^{1} \cdot\left(\gamma_{1}=\aleph_{0}, \gamma_{3}=\aleph_{\omega}, \gamma_{5}=\aleph_{\omega^{\omega}}\right.$, etc. See Jackson [3].) For $\lambda$ a projective ordinal, $\mu_{\lambda}$ denotes the unique supercompactness measure on $P_{\omega_{1}}(\lambda)$ (see Becker [1]). "Almost every" always refers to the measure $\mu_{\lambda}$.

Definition. Let $A \subset \mathcal{N}$ and let $\lambda$ be a projective ordinal.

(1) A $\lambda$-representation of $A$ is a function $F: P_{\omega_{1}}(\lambda) \rightarrow \mathcal{B}$ satisfying the following two properties:

(a) for all $S \in P_{\omega_{1}}(\lambda), F(S) \subset A$;

(b) for all $x \in A$, for almost every $S \in P_{\omega_{1}}(\lambda), x \in F(S)$.

(2) $A$ is $\lambda$-representable if there exists a $\lambda$-representation of $A$.

Main Theorem. Let $A \subset \mathcal{N}$. The following are equivalent:

(a) $A$ is $\boldsymbol{\Delta}_{2 n+1}^{1}$.

(b) $A$ is $\gamma_{2 n+1}$-representable.

The purpose of this paper is to prove the Main Theorem. The (a) $\Longrightarrow(b)$ direction is proved in $\S 2$, the $(\mathrm{b}) \Longrightarrow(\mathrm{a})$ direction in $\S 3$.

In Becker [2], it was proved that a set $A \subset \mathcal{N}$ is $\boldsymbol{\Sigma}_{2 n+2}^{1}$ iff it is $\boldsymbol{\delta}_{2 n+1}^{1}$-representable. In that paper, the Main Theorem of this paper was conjectured, and it was stated (without proof) that (b) $\Longrightarrow$ (a) holds. The only new result here is (a) $\Longrightarrow$ (b). We refer the reader to Becker [2] for more information on the subject of $\lambda$-representability.

The Main Theorem was presented at the Set Theory, Ergodic Theory and Banach Spaces Workshop, held at the University of Florida on May 5-11, 2007. The

Received by the editors January 7, 2010 and, in revised form, August 30, 2010

2010 Mathematics Subject Classification. Primary 03E15, 03 E60.

(C)2011 American Mathematical Society Reverts to public domain 28 years from publication 
applications of the Main Theorem, which were also presented at that meeting, will be published elsewhere.

\section{Proof OF (a) $\Longrightarrow$ (b)}

Lemma. Let $A \subset \mathcal{N}$ be $\boldsymbol{\Delta}_{2 n+1}^{1}$. There exists a tree, $T$, on $\omega \times \gamma_{2 n+1}$ such that $p[T]=A$ and such that for all $\sigma \in T, \sup \left\{\operatorname{rank}\left(T_{\sigma}[x]\right): x \notin p\left[T_{\sigma}\right]\right\}<\boldsymbol{\delta}_{2 n+1}^{1}$.

Proof. As $\delta_{2 n+1}^{1}=\gamma_{2 n+1}^{+}$, it will suffice to show that there exists an ordinal $\lambda<$ $\boldsymbol{\delta}_{2 n+1}^{1}$ and a tree $T$ on $\omega \times \lambda$ satisfying this lemma.

Let $\left\{\varphi_{m}\right\}_{m \in \omega}$ be a very good $\boldsymbol{\Delta}_{2 n+1}^{1}$-scale on the $\boldsymbol{\Delta}_{2 n+1}^{1}$ set $A$. Then there exists a $\lambda<\delta_{2 n+1}^{1}$ such that $\sup \left\{\varphi_{m}(x): m \in \omega, x \in A\right\}<\lambda$. Being very good means that

$$
\varphi_{m+1}(x) \leq \varphi_{m+1}(y) \Longrightarrow \varphi_{m}(x) \leq \varphi_{m}(y) .
$$

Such a scale exists, by Moschovakis [5, 4E.2 and 6C.4]. Let $T$ be the following tree on $\omega \times \lambda$ :

$$
\begin{aligned}
& \left\{\left(\left(a_{0}, \ldots, a_{j-1}\right),\left(\varphi_{0}\left(x_{0}\right), \ldots, \varphi_{j-1}\left(x_{j-1}\right)\right)\right) \in(\omega \times \lambda)^{<\omega}:(\forall i<j)\right. \\
& \left.\left(x_{i} \in A \text { and }\left(a_{0}, \ldots, a_{i}\right) \prec x_{i}\right) \text { and }(\forall i<j-1)\left(\varphi_{i}\left(x_{i+1}\right) \leq \varphi_{i}\left(x_{i}\right)\right)\right\} .
\end{aligned}
$$

Claim. Let $\sigma=\left(\left(a_{0}, \ldots, a_{j-1}\right),\left(\xi_{0}, \ldots, \xi_{j-1}\right)\right) \in T$. Then $p\left[T_{\sigma}\right]=\{x \in A$ : $\left(a_{0}, \ldots, a_{j-1}\right) \prec x$ and $\left.\varphi_{j-1}(x) \leq \xi_{j-1}\right\}$.

Assuming the Claim, we can quickly complete the proof. The $\sigma=\langle\rangle$ case of the Claim states that $p[T]=A$. Since $\left\{\varphi_{m}\right\}$ is a $\boldsymbol{\Delta}_{2 n+1}^{1}$-scale, the Claim shows that $p\left[T_{\sigma}\right]$ is a $\boldsymbol{\Delta}_{2 n+1}^{1}$ set. Therefore, $\left\{\operatorname{rank}\left(T_{\sigma}[x]\right): x \notin p\left[T_{\sigma}\right]\right\}$ is a $\boldsymbol{\Sigma}_{2 n+1}^{1}$ set of ordinals, hence bounded below $\boldsymbol{\delta}_{2 n+1}^{1}$.

All that remains to be proved is the Claim.

To prove it, fix $\sigma=\left(\left(a_{0}, \ldots, a_{j-1}\right),\left(\xi_{0}, \ldots, \xi_{j-1}\right)\right)$ in $T$.

Proof of $\supset$ direction. Let $x=\left(a_{0}, \ldots, a_{j-1}, a_{j}, \ldots\right) \in A$ be such that $\varphi_{j-1}(x) \leq$ $\xi_{j-1}$. Let $\left(x_{0}, \ldots, x_{j-1}\right)$ witness that $\sigma \in T$. Then

$$
\left(x,\left(\varphi_{0}\left(x_{0}\right), \ldots, \varphi_{j-1}\left(x_{j-1}\right), \varphi_{j}(x), \varphi_{j+1}(x), \varphi_{j+2}(x), \ldots\right)\right)
$$

is an infinite branch of $T_{\sigma}$, hence $x \in p\left[T_{\sigma}\right]$.

Proof of $\subset$ direction. Let $x \in p\left[T_{\sigma}\right]$. Trivially, $\left(a_{0}, \ldots, a_{j-1}\right) \prec x$. By definition of $T$, there exists a sequence $x_{0}, x_{1}, x_{2}, \ldots$ of elements of $A$ such that:

(i) for all $i, x_{i}\lceil(i+1)=x\lceil(i+1)$;

(ii) for all $i, \varphi_{i}\left(x_{i+1}\right) \leq \varphi_{i}\left(x_{i}\right)$;

(iii) $\varphi_{j-1}\left(x_{j-1}\right)=\xi_{j-1}$.

Since the scale is very good, (ii) implies that for all $i$, for all $k \leq i, \varphi_{k}\left(x_{i+1}\right) \leq$ $\varphi_{k}\left(x_{i}\right)$. So there exists a sequence of ordinals, $\eta_{0}, \eta_{1}, \ldots$, such that for all $k \in \omega$, for all sufficiently large $i, \varphi_{k}\left(x_{i}\right)=\eta_{k}$. By (i), $x_{i} \rightarrow x$. By definition of scale, $x \in A$, and for all $k, \varphi_{k}(x) \leq \eta_{k}$. In particular, $\varphi_{j-1}(x) \leq \eta_{j-1}$. Again using (ii) and the fact that the scale is very good, $\eta_{j-1} \leq \varphi_{j-1}\left(x_{j-1}\right)$. So, by (iii), $\varphi_{j-1}(x) \leq \xi_{j-1}$.

Proof of $(\mathrm{a}) \Longrightarrow$ (b) of the Main Theorem. Fix a $\boldsymbol{\Delta}_{2 n+1}^{1}$ set $A$ and let $T$ be a tree on $\omega \times \gamma_{2 n+1}$ satisfying the previous lemma. Since $\boldsymbol{\delta}_{2 n+1}^{1}$ is a regular cardinal, there is an ordinal $\xi, \gamma_{2 n+1} \leq \xi<\boldsymbol{\delta}_{2 n+1}^{1}$, such that:

for all $\sigma \in T$, for all $x \in \mathcal{N} \backslash p\left[T_{\sigma}\right], \operatorname{rank}\left(T_{\sigma}[x]\right)<\xi$. 
Fix a well ordering, $\unlhd$, of $\gamma_{2 n+1}$ of order-type $\xi$, and define $f: P_{\omega_{1}}\left(\gamma_{2 n+1}\right) \rightarrow \omega_{1}$ by

$$
f(S)=\text { order-type of }(\unlhd\lceil S) .
$$

For $S \in P_{\omega_{1}}\left(\gamma_{2 n+1}\right)$, let $T^{S}=T \cap(\omega \times S)^{<\omega}$, and let

$A^{S}=\left\{x \in \mathcal{N}:\right.$ It is not the case that $T^{S}[x]$ is wellfounded with

rank $\leq f(S)$, and for all $\sigma \in T^{S}[x]$, it is not the case that $T_{\sigma}^{S}[x]$

is wellfounded with rank exactly $f(S)\}$.

Note that $A^{S} \subset p\left[T^{S}\right]$. As $T^{S}$ is a countable tree and $f(S)$ is a countable ordinal, $A^{S}$ is a Borel set. Define $F: P_{\omega_{1}}\left(\gamma_{2 n+1}\right) \rightarrow \mathcal{B}$ to be the function $F(S)=A^{S}$. For any $S \in P_{\omega_{1}}\left(\gamma_{2 n+1}\right)$,

$$
F(S)=A^{S} \subset p\left[T^{S}\right] \subset p[T]=A .
$$

Let $\mu$ be $\mu_{\gamma_{2 n+1}}$. To complete the proof that $F$ is a $\gamma_{2 n+1}$-representation of $A$, all that remains to be shown is that for all $x \in A$, for $\mu$-almost every $S, x \in F(S)$.

Fix $x \in A$. Then $x \in p[T]$, so the fineness of $\mu$ implies that for almost every $S, x \in p\left[T^{S}\right]$. Assume, toward a contradiction, that for almost every $S, x \notin F(S)$. By definition of $F(S)$, this means that for almost every $S$, there exists a $\sigma^{S} \in T^{S}$ such that $T_{\sigma^{S}}^{S}[x]$ is wellfounded and $\operatorname{rank}\left(T_{\sigma^{S}}^{S}[x]\right)=f(S)$. The countable additivity and normality of $\mu$ imply that there is a fixed $\sigma$ such that $\sigma=\sigma^{S}$ for almost every $S$. Thus for almost every $S$, the ordinal $\operatorname{rank}\left(T_{\sigma}^{S}[x]\right)$ is the order-type of $\unlhd\lceil S$. For such $S$, let $g_{S}: T_{\sigma}^{S}[x] \rightarrow S$ be the canonical order-reversing $(\succeq$ to $\unlhd)$ function; then $g_{S}$ is onto. Let $g: T_{\sigma}[x] \rightarrow \gamma_{2 n+1}$ be the function $g(\tau)=g_{S}(\tau)$ for almost every $S$. Again using the elementary properties of $\mu$, we see that $g$ is a well-defined total function from $T_{\sigma}[x]$ onto $\gamma_{2 n+1}$, and it is the canonical order-reversing ( $\succeq$ to $\unlhd$ ) rank function. Hence

$$
\begin{aligned}
\operatorname{rank}\left(T_{\sigma}[x]\right) & =\text { order-type of } \unlhd \\
& =\xi .
\end{aligned}
$$

This contradicts the definition of the ordinal $\xi$.

$$
\text { 3. Proof OF (b) } \Longrightarrow(\mathrm{a})
$$

Fix a countable clopen basis, $\mathcal{C}$, for $\mathcal{N}$.

\section{Definition.}

(1) An $\infty$-Borel code is a triple $\mathcal{I}=(Z, T, h)$, where:

(a) $Z$ is a nonempty well orderable set;

(b) $T$ is a wellfounded tree on $Z$;

(c) $h$ is a function from the terminal nodes of $T$ into $\mathcal{C}$.

$Z$ is called the underlying set of $\mathcal{I}$.

(2) Given an $\infty$-Borel code $\mathcal{I}=(Z, T, h)$, for all $\sigma \in T$, we define a subset $B_{\sigma}\left(=B_{\sigma}(\mathcal{I})\right)$ of $\mathcal{N}$, by recursion on $T$, as follows:

(i) $B_{\sigma}=h(\sigma)$, if $\sigma$ is a terminal node of $T$.

(ii) $B_{\sigma}=\bigcap\left\{B_{\sigma \frown s}: s \in Z\right.$ and $\left.\sigma \frown s \in T\right\}$, if $\sigma$ is not a terminal node and length $(\sigma)$ is even.

(iii) $B_{\sigma}=\bigcup\left\{B_{\sigma \frown s}: s \in Z\right.$ and $\left.\sigma \frown s \in T\right\}$, if $\sigma$ is not a terminal node and length $(\sigma)$ is odd.

We say that $B_{\langle\rangle}$is the subset of $\mathcal{N}$ encoded by $\mathcal{I}$ or that $\mathcal{I}$ is a code for $B_{\langle\rangle}$. 
For $S \in P_{\omega_{1}}(\lambda)$, we view $S^{\omega}$ as a Polish space, with the discrete topology on $S$ and the product topology on $S^{\omega}$. For $p \in S^{<\omega}, N_{p}^{S}$ denotes the neighborhood of the space $S^{\omega}$ consisting of all infinite sequences which extend $p$.

The following lemma is due to Kechris-Woodin [4. This lemma gives a system of "generic codes" for uncountable ordinals.

Lemma. There exists a $\boldsymbol{\Delta}_{2 n+1}^{1}$ set $W \subset \mathcal{N}$, a surjection $\psi: W \rightarrow \gamma_{2 n+1}, \psi: w \mapsto$ $|w|$, such that the prewellordering of $W$ induced by $\psi$ is $\boldsymbol{\Delta}_{2 n+1}^{1}$, and there exists a Lipschitz function $G:\left(\gamma_{2 n+1}\right)^{\omega} \rightarrow W^{\omega}$ such that for almost every $S \in P_{\omega_{1}}\left(\gamma_{2 n+1}\right)$, for a comeager set of $f \in S^{\omega}$, for all $i \in \omega,\left|(G(f))_{i}\right|=f(i)$.

Proof of (b) $\Longrightarrow$ (a) of the Main Theorem. Fix a $\gamma_{2 n+1}$-representation

$$
F: P_{\omega_{1}}\left(\gamma_{2 n+1}\right) \rightarrow \mathcal{B}
$$

for $A$. For every $S \in P_{\omega_{1}}\left(\gamma_{2 n+1}\right), F(S)$ is a Borel set; hence there exists an $\infty$-Borel code, $\mathcal{I}$, for $F(S)$ with underlying set $\omega$, i.e., $\mathcal{I}=(\omega, T, h)$. Note that $\mathcal{I}$ "is" a real.

Let $\mu$ be $\mu_{\gamma_{2 n+1}}$. By Becker [1, 3.2], there is a set $\mathcal{S} \subset P_{\omega_{1}}\left(\gamma_{2 n+1}\right)$ with $\mu(\mathcal{S})=1$ such that $\mathcal{S}$ and the set

$$
\{(S, \mathcal{I}): S \in \mathcal{S} \text { and } \mathcal{I}=(\omega, T, h) \text { is an } \infty \text {-Borel code for } F(S)\}
$$

are projective sets. Using the previous lemma and projective uniformization, there exists a function $f \mapsto \mathcal{I}^{f}$ with domain $\left(\gamma_{2 n+1}\right)^{\omega}$ such that for $\mu$-almost every $S$, for a comeager set of $f \in S^{\omega}, \mathcal{I}^{f}=\left(\omega, T^{f}, h^{f}\right)$ is an $\infty$-Borel code for the Borel set $F(S)$. For any such $f, T^{f}$ is a well founded tree on $\omega$; let $\rho^{f}: T^{f} \rightarrow \omega_{1}$ be the rank function.

Let $Z=\left(\gamma_{2 n+1}^{<\omega} \times \omega\right)$. We define a tree $\widetilde{T}$ on $Z$. Let $\widetilde{T}=$

$$
\begin{aligned}
& \left\{\left(\left(p_{0}, m_{0}\right),\left(p_{1}, m_{1}\right), \ldots,\left(p_{k-1}, m_{k-1}\right)\right): \forall j<k-1,\right. \\
& \quad p_{j} \prec p_{j+1} \text { and } \forall j<k \text {, for a.e. } S, \exists \xi<\omega_{1} \text { such that for a comeager-in- } N_{p_{j}}^{S} \text { set } \\
& \text { of } \left.f \in S^{\omega},\left(m_{0}, m_{1}, \ldots, m_{j-1}\right) \in T^{f} \text { and } \rho^{f}\left(m_{0}, m_{1}, \ldots, m_{j-1}\right)=\xi\right\} .
\end{aligned}
$$

We next define a partial function $\tilde{h}$ from the terminal nodes of $\widetilde{T}$ into $\mathcal{C}$. Let $\sigma=\left(\left(p_{0}, m_{0}\right), \ldots,\left(p_{k-1}, m_{k-1}\right)\right)$ be a terminal node. Then

$$
\tilde{h}(\sigma)=\left\{\begin{array}{c}
M, \text { if for a.e. } S, \text { for a } \\
\text { comeager-in- } N_{p_{k-1}}^{S} \text { set of } \\
f \in S^{\omega}, \sigma^{*}=\left(m_{0}, \ldots, m_{k-1}\right) \\
\text { is a terminal node of } \\
T^{f} \text { and } h^{f}\left(\sigma^{*}\right)=M ; \\
\text { undefined otherwise. }
\end{array}\right.
$$

Let $T=\{\sigma \in \widetilde{T}: \exists \tau \succeq \sigma$ such that $\tau \in(\widetilde{T} \cap \operatorname{dom}(\tilde{h}))\}$. Let $h=\tilde{h}\lceil T$ and let $\mathcal{I}=(Z, T, h)$.

It is obvious that $Z$ is a nonempty well-orderable set, that $T$ is a tree on $Z$ and that $h$ is a total function from the terminal nodes of $T$ into $\mathcal{C}$. Some routine supercompactness and Baire-category arguments establish the following two facts. First, that $T$ is wellfounded. Hence $\mathcal{I}=(Z, T, h)$ is an $\infty$-Borel code. Second, by induction on the wellfounded tree $T$ : let $\sigma=\left(\left(p_{0}, m_{0}\right), \ldots,\left(p_{k-1}, m_{k-1}\right)\right)$ and $\sigma^{*}=\left(m_{0}, \ldots, m_{k-1}\right)$, where $k$ is even (respectively, odd); for all $x \in \mathcal{N}, x \in B_{\sigma}(\mathcal{I})$ iff for almost every $S$, for a comeager-in- $N_{p_{k-1}}^{S}$ (respectively, nonmeager-in- $N_{p_{k-1}}^{S}$ ) 
set of $f \in S^{\omega}, x \in B_{\sigma^{*}}\left(\mathcal{I}^{f}\right)$. Therefore, for any $x \in \mathcal{N}, x \in B_{\langle\rangle}(\mathcal{I})$ iff for almost every $S$, for a comeager set of $f \in S^{\omega}, x \in B_{\langle\rangle}\left(\mathcal{I}^{f}\right)$. Recall that $\mathcal{I}^{f}$ was chosen to be an $\infty$-Borel code for $F(S)$, where $S=\operatorname{Image}(f)$. This means that $x \in B_{\langle\rangle}(\mathcal{I})$ iff for almost every $S, x \in F(S)$. As $F$ is a $\gamma_{2 n+1}$-representation for $A$, by definition of $\gamma_{2 n+1}$-representation, $B_{\langle\rangle}(\mathcal{I})=A$.

We have thus shown that $A$ is encoded by an $\infty$-Borel code with underlying set $Z$. Since $Z$ has cardinality $\gamma_{2 n+1}, A$ is a $\gamma_{2 n+1}^{+}$-Borel $\left(=\delta_{2 n+1}^{1}\right.$-Borel $)$ set. By a theorem of Martin (see Moschovakis [5, 7D.9]), every $\boldsymbol{\delta}_{2 n+1}^{1}$-Borel set is $\boldsymbol{\Delta}_{2 n+1}^{1}$.

\section{REFERENCES}

[1] H. Becker, AD and the supercompactness of $\aleph_{1}$, Journal of Symbolic Logic 46 (1981), 822-842. MR.641495 (83b:03061)

[2] H. Becker, Representing projective sets as unions of Borel sets, Proceedings of the American Mathematical Society 123 (1995), 883-886. MR.1224612 (95d:03086)

[3] S. Jackson, Structural consequences of $A D$, in Handbook of Set Theory, vol. 3, M. Foreman and A. Kanamori (eds.), Springer, 2010.

[4] A.S. Kechris and W.H. Woodin, Generic codes for uncountable ordinals, partition properties, and elementary embeddings, in: A.S. Kechris, B. Löwe and J.R. Steel (eds.), Games, Scales and Suslin Cardinals, Lecture Notes in Logic, 31, Cambridge University Press, 2008, pp. 379-397. MR 2463619

[5] Y.N. Moschovakis, Descriptive Set Theory, North-Holland Publishing Company, 1980. MR561709 (82e:03002)

4840 Forest Drive, Suite 6-B, PMB 128, Columbia, South Carolina 29206

E-mail address: hsbecker@hotmail.com 